\title{
PROBLEMAS DE AUTORÍA EN LA TRADUCCIÓN LATINA MEDIEVAL DEL LIBRO VI DEL DE SIMPL. MED. FAC. DE
}

GALENO $^{1}$

\author{
MARINA DÍAZ MARCOS \\ Escuela de Traductores de Toledo \\ marina.diaz@uclm.es \\ ORCID: 0000-0002-5743-8068
}

\section{RESUMEN}

En el Occidente medieval apenas existían versiones en latín de las obras científicas griegas hasta el siglo XII, cuando nace la traducción científica propiamente dicha. En el ámbito de la Escuela de Traductores de Toledo una de las figuras más destacadas fue Gerardo de Cremona (s. XII), quien tradujo del árabe al latín el De simplicium medicamentorum facultatibus I-V/VI de Galeno, un tratado de farmacología compuesto por once libros. La complejidad de la transmisión del texto y los escasos estudios sobre las traducciones de textos médicos realizadas por Gerardo han supuesto un obstáculo en el análisis de la obra. Uno de los libros que más problemas suscita es el VI, ya que se conserva solo en 5 manuscritos (los libros I-V en 55) y se duda de su autoría, puesto que Gerardo nunca firmaba sus obras.

El objetivo de esta propuesta es defender la autoría de dicho libro por parte de Gerardo mediante su estudio y posterior comparación con los otros libros, sí atribuidos a él. El resultado mostrará que el traductor es el mismo y que el libro VI fue publicado con posterioridad a los cinco primeros.

PALABRAS CLAVE: De simplicium medicamentorum facultatibus, Gerardo de Cremona, Galeno, libro VI, autoría.

\section{PROBLEMS OF AUTHORSHIP IN THE MEDIEVAL LATIN TRANSLATION OF BOOK VI OF GALEN'S DE SIMPL. MED. FAC.}

\section{ABSTRACT}

In the Medieval Occident there were not much Latin versions of scientific Greek works until the 12th century, when scientific translation was truly born. At this time, Gerard of Cremona, one of the most important personalities of the Toledo School of Translators, translated the eleven books of Galen's pharmacological work De simplicium medicamentorum facultatibus I-V/VI from Arabic into Latin. The study of this treatise has always been quite difficult due to the complexity in the transmission of the text and the scarce information about the translations of medical texts made by Gerard. Book VI is the most problematic regarding its authorship, given that it is only preserved in 5 manuscripts (55 for books I-V) and without the signature of the translator.

The aim of this article is to defend Gerard's authorship of book VI by means of its study and comparison to the other books, already attributed to him. The result will show that, in fact, the

${ }^{1}$ Este trabajo se inscribe dentro de las líneas de investigación del proyecto «Galenus Latinus: Recuperación del Patrimonio de la Medicina Europea II» (FFI2016-77240-P) del grupo I+D Interpretes Medicinae (INTERMED), que lidera la Red de Excelencia «Opera Medica: Recuperación del Patrimonio Textual Grecolatino de la Medicina Europea II» (RED2018-102781-T). 
translator is the same and that book VI was published afterwards.

KEYWORDS: De simplicium medicamentorum facultatibus, Gerard of Cremona, Galen, book VI, authorship.

\section{LA ESCUELA DE TRADUCTORES DE TOLEDO MEDIEVAL Y LA LABOR TRADUCTORA DE GERARDO DE CREMONA}

La Edad Media no fue una época de creación de nuevos conocimientos, sino más bien de transmisión del saber antiguo. Las traducciones al latín de textos científicos árabes y griegos (a través del árabe) fueron un método de trabajo extendido en esta época por diferentes puntos de Europa (Montero Cartelle 2010: 33), especialmente en el siglo XII, cuando nace la traducción propiamente dicha. Toledo, dotada de una población de gran diversidad lingüística, es famosa por ser el principal centro de traducción de textos científicos y filosóficos del árabe al latín, los cuales cambiaron el saber científico del momento al ser introducidos en otros centros de enseñanza europeos (Gil 1985: 15-17).

En el ámbito de la Escuela de Traductores de Toledo medieval, Gerardo de Cremona fue el traductor más prolífero de obras científicas en el siglo XII. A diferencia de otros traductores, llevó a cabo una búsqueda de diferentes manuscritos que pudieran contener el texto, dando lugar a trabajos muy literales resultado de la comparación de copias fidedignas (Jacquart 1991: 185-8).

Esos tratados científicos, de frecuente origen griego, antes de tener una versión en lengua latina, habían pasado, como ya hemos indicado, por un eslabón intermedio: el árabe. Una obra de este tipo es el De simplicium medicamentorum facultatibus I-V/VI (a partir de ahora De simpl.) de Galeno. Está compuesta por once libros, los cuales se han transmitido de manera bipartita desde los primeros testimonios en griego (salvo en algunos manuscritos recientes), llegando a generar una tradición propia: por un lado, los libros $\mathrm{I}-\mathrm{V}$, correspondientes a la teoría (propiedades del cuerpo y medicamentos simples), y, por otro, los libros VI-XI, la parte práctica (cualidades de remedios simples particulares) (Petit 2010: 147). La problemática de la traducción latina de Gerardo viene dada, como se indica en el título de este trabajo, por la autoría del libro VI.

\section{LA TRANSMISIÓN DEL DE SIMPL. DE GALENO}

En la tradición manuscrita de este tratado existen testimonios griegos bastante antiguos, a diferencia de otras obras, pero también traducciones en siríaco, árabe y latín, ya sea por tradición directa o indirecta (Garofalo 1985: 317). Antes de ser vertido a la lengua latina, el De simpl. pasó por diversas fases. En primer lugar, en el siglo VI, Sergius de Rēš 'Aynā tradujo la obra del griego al siríaco; Hunayn ibn Ishạā, en el siglo IX, hizo lo mismo. Sin embargo, este, del mismo modo que Al-Bițrīq y Hubaiš, tradujo más tarde la obra al árabe a partir o bien del siríaco o 
bien del griego, dando lugar a un panorama en el que varias traducciones árabes del tratado circulaban en Oriente al mismo tiempo. Más adelante, Gerardo de Cremona llevó a cabo en el siglo XII una traducción muy literal de los cinco primeros libros (y quizás del VI) del árabe al latín (Petit 2016: 2, 18). Dos siglos más tarde, Niccolò de Reggio realizó una nueva traducción latina, pero en este caso a partir del griego y de los once libros completos (Petit 2010: 148; 2013: 1069). Fue más conocida su segunda parte, ya que ayudó a la hora de completar el trabajo de Gerardo.

La transmisión conjunta de las traducciones de Gerardo (libros I-V y ¿VI?) y Niccolò (VII-XI) dio lugar a la publicación de una edición en el año 1490 a manos de Diomedes Bonardus, la única por la que fue conocido el tratado hasta comienzos del siglo XVI (Fortuna 2005: 472-3, 2012: 394-5, Díaz Marcos 2020: 2401).

No obstante, este tratado ha sido mal comprendido hasta la publicación de las primeras ediciones humanistas (Petit 2013: 1077-8, Díaz Marcos 2020: 238-9): la edición griega de Aldo Manuzio, la Aldina (1525); la edición latina (1530) de Theodoricus Gerardus Gaudanus (Calà 2013: 1105-7) realizada a partir del griego. Surgió como reacción de algunos estudiosos que, rechazando las traducciones árabo-latinas, se afanaron en depurar los textos antiguos consultando las versiones griegas de las obras y recuperando el latín de época clásica. Esta obra, que aseguró la difusión del tratado en el Renacimiento, fue publicada de manera póstuma por Johannes Sturn (1507-1589), ya que Gaudanus la había escrito para uso privado.

Tras esta última, se fueron sucediendo diversas ediciones de la obra, a veces simplemente reimpresiones, hasta la de Kühn, de principios del siglo XIX, que recoge todas las obras de Galeno en 20 volúmenes.

\section{EL LIBRO VI DEL DE SIMPL.: PROBLEMAS DE AUTORÍA}

El libro VI en latín está formado por una introducción seguida de un listado de 145 simples (23 menos que en las otras traducciones) acompañados de su correspondiente descripción: propiedades de las plantas basadas en las cuatro cualidades básicas (cálido, húmedo, seco y frío) que definían la materia orgánica y las enfermedades o dolencias contra las que se usaban en todos los aparatos del cuerpo humano.

Algunos investigadores como Richard Lemay, Danielle Jacquart y Charles Burnett han rechazado la autoría de este libro por parte de Gerardo, apoyándose en los siguientes puntos que recoge Petit (2013: 1069-70):

1. Gerardo nunca escribía su nombre en los trabajos que realizaba. Hay dos manuscritos que lo mencionan como autor al comienzo del libro I, en una rúbrica, pero ninguno contiene la traducción del libro vi: el Vat. Pal. lat. 1092, f. 22r (final s. XIII), y el Kraków 800, f. 1r (1279). También en el Par. lat. 9331, f. 202r (esta vez sí con el libro VI), aparece el nombre de 
Gerardo, pero en una glosa escrita en el margen por otra mano diferente a la del texto.

2. La parte práctica apenas era conocida en el Occidente medieval a excepción del libro VI.

3. No existe ningún manuscrito latino que ofrezca una traducción completa de los once libros a partir del árabe.

Es importante también mencionar que no se sabe con certeza qué manuscrito(s) árabe(s) pudo utilizar Gerardo como modelo(s) para la traducción de la obra, ni siquiera si contenía(n) la versión árabe de Ḥunayn, la más extendida. Atendiendo al número de arabismos presentes en el texto latino y a la técnica traductora de aquel (Ventura 2019: 403), podemos afirmar que, gracias a sus conocimientos de árabe, seguía fielmente la sintaxis del texto escrito en esta lengua y acudía más a la transliteración («préstamo léxico») que a la traducción de los términos. Cuando se trataba de un fitónimo, generalmente utilizaba este recurso y después ofrecía el sinónimo latino precedido de la locución id est.

Para la edición latina del libro VI me he servido de los siguientes manuscritos ${ }^{2}$ árabes para realizar correcciones, algunos de los cuales están siendo también utilizados en el corpus trilingüe online (en proceso) del proyecto HUNAYNNET: la versión de Ḥunayn (Derenbourg 1941: 3-4, Diels 1905-1907: 97): El Escorial, Real Biblioteca del Monasterio de San Lorenzo de El Escorial, ar. 793, ff. 90v-110r; y 794, ff. 1v-22r; la de Hubaiš (Garofalo 1985: 318): Florencia, Biblioteca Medicea Laurenziana, Orient. 193, ff. 100r-124r; la de Al-Bițrīq (Ventura 2019: 402): Estambul, Saray Ahmet III, 2083, ff. 127r-152v.

Es oportuno, pues, acercarse al texto latino para examinar la autoría del libro VI. Frente a los 55 manuscritos datados hasta el momento que conservan la traducción de Gerardo de los cinco primeros libros, del VI solo hay cinco, como he podido comprobar tras el estudio de los catálogos de H. Diels (1905-1907: 97), R. J. Durling (1967: 471) y S. Fortuna (2008: online), continuadora del trabajo de este. Desgraciadamente, dichos manuscritos son posteriores a la fecha de la traducción de Gerardo:

\footnotetext{
VP1 Città del Vaticano, Biblioteca Apostolica Vaticana, Pal. lat. 1094.

(2 ${ }^{\mathrm{a}}$ mitad s. XIV). L. VI: ff. 549r-563r.

$\mathrm{V}^{1}$ Città del Vaticano, Biblioteca Apostolica Vaticana, Vat. lat. 2376.

(1ª mitad s. XIV). L. VI: ff. 89r-90r.

V2 Città del Vaticano, Biblioteca Apostolica Vaticana, Vat. lat. 2385.

(1aㅡ mitad s. XIV). L. VI: ff. 125v-135r.

L1 Leipzig, Universitätsbibliothek, 1136.

(1 ${ }^{\text {a }}$ mitad s. XIV). L. VI: ff. 239v-250v.

$\mathrm{P}^{1}$ Paris, Bibliothèque Nationale de France, Par. lat. 9331.

(Final s. XIV). L. VI: ff. 256r-266r.
}

\footnotetext{
2 Son bastante tardíos, de los siglos XIII y XIV.
} 
Podemos establecer la existencia de dos ramas diferentes para estos manuscritos, agrupación que se repite tanto para la transmisión de los libros I-V como para el VI. Veámoslas con relación a este último:

1. En una encontramos solo el $\mathrm{VP}^{1}$, en el que el libro VI aparece separado de los cinco primeros (ff. 31v-85v) dentro del códice y escrito por un copista diferente. Ofrece una versión más reducida del texto, con 25 simples menos que el resto de manuscritos, pero mejor transliterados del árabe al latín. Su sintaxis es más sencilla, no coincidente en muchos casos con la del texto árabe, pero conserva pasajes que omiten el resto de testimonios. He podido comprobar, además, que no sirvió de modelo a la edición de 1490. Incluye al final un listado alfabético de simples redactado por el mismo copista del libro, pero con el nombre latino del simple en primer lugar. Por ejemplo, en lugar de karm albarri, id est, uitis siluestris (lo esperable), encontramos uitis siluestris dicitur kemal. ${ }^{3}$

2. En la segunda encontramos el resto de los testimonios: el $\mathrm{V}^{1}$ conserva solo la parte introductoria de la obra; el $\mathrm{V}^{2}$ añade en rúbricas el nombre latino del simple en caso Ablativo precedido de la preposición de; el L ${ }^{1}$ presenta en el margen el nombre griego de la planta con caracteres latinos; el $\mathrm{P}^{1}$ vio completadas sus lagunas con la ayuda de hasta tres manos diferentes.

Ofrecemos algunos ejemplos ${ }^{4}$ que apoyan la existencia de dos ramas para el libro VI:

1. El nombre de Hunayn aparece al comienzo del libro en todos los testimonios excepto en el VP. En el $\mathrm{V}^{1}$ y el $\mathrm{L}^{1}$ suponemos que debería haberse escrito en el espacio en blanco destinado a la rúbrica. Es probable, pues, que el texto de Hunayn sí sirviera de modelo al traductor latino, al menos para este libro.

2. En la parte conservada en $\mathrm{el} \mathrm{V}^{1}$ encontramos pasajes en los que todos los manuscritos omiten alguna parte del texto por un saut du même au même, excepto el $\mathrm{VP}^{1}$. Me refiero a la parte destaca en negrita en este ejemplo, conservada también en el texto árabe: ${ }^{5}$

(...) in tractatu primo in quo locuti fuimus de aqua et aceto et in tractatu secundo quando locuti fuimus de oleo et oleo rosaceo] in quo loquti sumus de aqua et aceto et in tractatu secundo quando loquti sumus de oleo et oleo rosaceo $V P^{1}$ : in quo locuti fuimus de oleo et oleo rosaceo $L^{1} P^{1} V^{1} v^{2}$.

3. En cambio, el $\mathrm{VP}^{1}$ suele cambiar algunos términos por sinónimos o presentar una sintaxis más breve, no coincidente con la del texto árabe:

\footnotetext{
${ }^{3}$ Kemal corresponde a la lectura del simple sin editar.

4 Todos han sido tomados del texto de mi edición crítica.

في المقالة الأولى من هذا الكتاب حيث بحثثا عن الماء والخلّ وفي المقالة الثانية أيضاً لما بحثثا عن الزيت وعن دهن الورد 5
} 
lumbricos $\left.{ }^{6}\right]$ uermes $V P^{1}$.

(...) res aliquas narratione bona, sed nullus hominum narrauit omnes nisi solummodo iste] sicut et ipse fecit $V P^{1}$.

4. Dentro del segundo grupo, los manuscritos también presentan algunas variantes propias que demuestran que ninguno sirvió de modelo a otro, sino que proceden de uno varios testimonios no conservados:

(...) ex sucis plantarum et quedam ex gummis et quedam] om. $V^{1}$ : ex succis plantarum et quedam ex gummis et quedam $V P^{1}$.

O los siguientes ejemplos, donde el $\mathrm{V}^{1}$ ya no conserva texto:

(...) hoc nomen est deriuatum a nomine regionis in qua crescit et est nomen terre Centonia] hoc namque est deriuatum a nomine regionis in qua crescit et est nomen terre Centonia $P^{1}:$ om. $V^{2}$.

(...) oleum saporiferum, quod est factum ex oliuis maturis, sed oleum, quod est extractum ex oliuis immaturis] oleum saporiferum quod est factum ex oliuis immaturis $L^{1}$ : oleum saporiferum quod est factum de oliuis maturis sed oleum quod est factum ab oliuis immaturis $P^{1}$ : oleum saporiferum quod est factum de oliuis maturis sed oleum quod est tractum ex oliuis immaturis $V P^{1}$.

Para poder defender la autoría de Gerardo con respecto al libro VI, hay una serie de recursos utilizados en la traducción que se van a repetir a lo largo de todos los libros. Encontramos, por ejemplo, bastantes transliteraciones de términos árabes al latín, sobre todo de simples: zaiton, ${ }^{7}$ id est, 'oliua'; o la traducción literal de palabras, como el topónimo Fonte Zeruina ${ }^{8}$ (o Ceruina), que se presenta como un calco del árabe عين زربه / Ayn Zarba/. En cuanto a la aparición del orden de las palabras, ya hemos visto en el ejemplo 2 que sigue literalmente la sintaxis del texto árabe.

Según Jacquart (1989: 113), otra característica de la técnica de Gerardo es la repetición de una serie de expresiones que lo identifican como traductor: secundum quod narrauimus, donec, non... nisi..., etc. Sin embargo, aún quedan muchas obras del cremonense por editar, por lo que no podemos hablar de la existencia de un estudio propiamente dicho sobre su técnica de traducción. Por ejemplo, si comparamos los libros I y VI, vemos que en ambos se repiten también ciertas estructuras, que, no obstante, no coinciden totalmente con las que indica Jacquart: uerumtamen, uero, immo, non... nisi..., propterea quia/quod, etc. También señala esta que el Cremonense siempre traduce de la misma manera ciertas locuciones árabes, como, por ejemplo, وذلك أنّا (wa dālika anna), que en latín sería quod est quia. Sin embargo, en el De simpl. no siempre es así, ya que esta expresión la encontramos como illud est quia/quoniam (L. I) y hoc est quia/quoniam (L. VI). Como afirma McVaugh (2009: 107-111), es probable que las variantes de esta estructura sean fruto del momento en que Gerardo realizó cada traducción, con

\footnotetext{
${ }^{6} \mathrm{El} \mathrm{V}{ }^{1}$ no conserva este pasaje.

7 الزَيُُون /al-zaītuwn/. Las variantes de los mss. latinos son: zarton $\mathrm{L}^{1}$, çaiton $\mathrm{P}^{1} \mathrm{y}$ çayton $\mathrm{V}^{2}$.

${ }^{8}$ La ciudad de Anazarba.
} 
el consecuente cambio o mejora de su técnica, y no de la existencia de dos traductores.

Por último, advertimos una serie de errores que llevan a pensar que la intención de Gerardo era la de traducir toda la obra y revisarla posteriormente, pero, por razones desconocidas, no terminó su trabajo. ${ }^{9}$ Por ejemplo, el nombre propio árabe latinizado como Abrucalis, que hace referencia a Empédocles, en el De simpl. es Herófilo. El error es fácilmente comprensible si tenemos en cuenta que los manuscritos árabes carecían de signos diacríticos $\mathrm{y}$, por tanto, podían llevar a confusión (en este caso, - اير-/Ayr-/10 por - ابر/Abr-/). Gerardo, pues, transliteró los nombres propios de médicos griegos en lugar de traducirlos al latín. Otros ejemplos son los siguientes (en gen. sing.):

L. I: Praxágoras como Faraxagori ${ }^{11}$ > فر اكساغورس/Frāksāgwrs/.

L. VI: Cratevas como Farataus ${ }^{12}>$ فراطورس/Frāṭawws/. ${ }^{13}$

\section{CONSIDERACIONES FINALES}

La historia de las traducciones del De simpl. de Galeno constituye un componente importante de la historia de la farmacología. En los cinco manuscritos que conservan la traducción latina, el segundo grupo es el que sigue de forma más literal la sintaxis del texto árabe, mientras que $\mathrm{VP}^{1}$ conserva pasajes que los otros omiten. En cuanto a los simples, $\mathrm{VP}^{1}$ contiene un menor número, pero suele presentar los nombres de los mismos menos deformados con respecto al original.

Todavía es pronto para establecer un estudio completo sobre la técnica de traducción y uso del lenguaje del cremonense, ya que quedan muchas obras suyas por editar y estudiar. Sin embargo, sí podemos afirmar que el traductor de los seis libros del De simpl. en latín es el mismo debido a las semejanzas y errores compartidos entre ellos.

La principal conclusión que planteamos con relación a la autoría y carácter inacabado del libro VI es que, por la razón que fuese, Gerardo no finalizó la traducción completa de la obra. Es sabido que sus socii publicaron el listado de obras traducidas por él al final del Tegni (Jacquart 1989: 111), entre cuyos títulos se puede leer: Liber $G$. de simplici medicina tractatus $V$. Por tanto, desconocían la existencia del borrador del libro VI. Cuando este fue descubierto, los cinco primeros libros ya habían empezado a ser copiados, lo que explicaría que la difusión de estos junto con el vi fuera menor y concentrada en un único grupo de

\footnotetext{
9 Ya hemos señalado más arriba que la versión latina conserva 23 simples menos que la griega, siríaca y árabe.

${ }^{10}$ Ayr- > Her-.

${ }^{11}$ Gen. sing. en $-i$ en lugar de en $-(a) e$.

12 Nombre transliterado directamente del árabe que no ha adoptado la terminación del Gen. sing. latino.

13 Variante de los manuscritos árabes con respecto a la forma correcta قر اطاوس /Qrāṭāws/.
} 
manuscritos. $^{14}$ Esta idea se vería reforzada por el explicit del libro VI del manuscrito $L^{1}$, en el que el copista era consciente de que dicho libro era una traducción de Gerardo y que no había sido incluido en la lista ofrecida por los socii: Explicit sextus liber Galieni De simplicibus medicinis noviter inventus.

\section{BIBLIOGRAFÍA}

CALÀ, I. (2013), «Theodoricus Gerardus Gaudanus traduttore di Galeno», Medicina nei secoli, 25/3, 1105-16.

DerenbourG, H. (1941), Les manuscripts arabes de l'Escurial. Vol. 5, París, Libraire Orientaliste Paul Geuthner.

DÍAZ MARCOS, M. (2020), «La tradición latina renacentista del De simplicium medicamentorum facultatibus de Galeno», en Acta Conventus Neo-latini Albasitensis. Proceedings of the Seventeenth International Congress of Neo-Latin Studies (Albacete 2018), Schaffenrath, F. y Santamaría Hernández, Ma․ T. (eds.), Leiden, Koninklijke Brill NV, 236-247.

DIELS, H. (1905-1907), Die Handschriften der antiken Ärzte. I. Hippocrates und Galenos, vol. 1, Berlín, Verlag der königlichen Akademie der Wissenschaften.

DURLING, R. J. (1967), «Corrigenda and addenda to Diel's Galenica I. Codices Vaticani», Traditio: Studies in Ancient and Medieval History, Thought and Religion, 23, 461476.

FORTUNA, S. (2005), «Galeno latino, 1490-1533», Medicina nei secoli, 17/2, 469-506.

ForTunA, S. Galeno. Catalogo delle traduzioni latine [en línea]. Università Politecnica delle Marche, 2008 [Consulta: 19 mayo 2020]. Disponible en: <www.galenolatino.com>.

FORTUNA, S. (2012), «The Latin Editions of Galen's Opera omnia (1490-1625) and their prefaces, Early Science and Medicine, 17, 391-412.

GAROFALO, I. (1985), «Un sondaggio sul De simplicium medicamentorum facultate di Galeno», en Studi arabo-islamici in onore di Roberto Rubinacci nel suo settantesimo compleanno, Sarnelli Cerqua, C. (ed.), vol. 1, Nápoles, Istituto Universitario Orientale, 317-325.

GIL, J. S. (1985), La Escuela de Traductores de Toledo y los colaboradores judíos, Toledo, Instituto Provincial de Investigaciones y Estudios Toledanos.

KESSEL, G. HUNAYNNET. Transmission of classical scientific and philosophical literature from Greek into Syriac and Arabic [en línea]. Österreichische Akademie der Wissenschaften, 2016 [Consulta: 19 mayo 2020]. Disponible en: $<$ https://hunaynnet.oeaw.ac.at>.

JACQUART, D. (1989), «Remarques préliminaires a une étude comparée des traductions médicales de Gérard de Crémone», en Traduction et traducteurs au Moyen Ấge. Actes du colloque international du CNRS organise à Paris, Institut de Recherche et d'Histoire des Textes, les 26-28 mai 1986, Contamine, G. (ed.), París, CNRS, 109-118. JACQUART, D. (1991), «L'école des traducteurs», en Tolède, XIIe-XIIIe. Musulmans, chrétiens et juifs: le savoir et la tolérance, Cardaillac, L. (ed.), París, Autrement, 177-191.

\footnotetext{
${ }^{14} \mathrm{El} \mathrm{VP}{ }^{1}$, como ya hemos señalado, parece un caso aislado, una copia realizada con posterioridad a la de los cinco primeros libros como demuestran su sintaxis más sencilla, la omisión (¿intencionada?) de algunos simples y la letra del copista.
} 
MCVAUGH, M. (2009), «Towards a stylistic grouping of the translations of Gerard of Cremona», Mediaeval studies, 71, 99-112.

Montero CArtelle, E. (2010), Tipología de la literatura médica latina: Antigüedad, Edad Media, Renacimiento, Oporto, Gabinete de Filosofia Medieval, Facultade de Letras.

PETIT, C. (2010), «La tradition manuscrite du traité des Simples de Galien. Editio princeps et traduction annotée des chapitres 1 à 3 du libre I», en Histoire de la tradition et édition des médecins grecs - Storia della tradizione e edizione dei medici greci. Serie Collectanea, 27, Boudon-Millot, V., Jouanna, J., Garzya, A. y Roselli, A. (eds.), Nápoles, D' Auria, 143-165.

PETIT, C. (2013), «La tradition latine du traité des Simples de Galien: étude préliminaire», Medicina nei Secoli, 25/3, 1063-1090.

PETIT, C. (2016), «La tradition manuscrite du Livre VI du traité des Simples de Galien», en Atti del VII Colloquio internazionale sull'Ecdotica dei testi medici greci (Procida 11-13 giugno 2013), Boudon-Millot, V., Garzya, A., Jouanna, J. y Roselli, A. (eds.), Nápoles, D'Auria, 1-20.

VenturA, I. (2019), "Galenic Pharmacology in the Middle Ages: Galen's On the Capacities of Simple Drugs and its Reception between the Sixth and Fourteenh Century», en Brill's Companion to the Reception of Galen, Bouras-Vallianatos, P. y Zipser, B. (eds.), Leiden y Boston, Brill, 393-433. 
